\title{
DELAMINATION ANALYSIS BY DAMAGE MECHANICS: SOME APPLICATIONS
}

\author{
L. Daudeville*, O. Allix ${ }^{\dagger}$ and P. Ladevèze \\ Laboratoire de Mécanique et Technologie, ENS de Cachan/CNRS/Univ. P. et M. Curie, 61 av. \\ du Président Wilson, 94235 Cachan Cedex, France
}

(Received 29 June 1994; accepted 20 September 1994)

\begin{abstract}
Delamination is a phenomenon which involves complex degradation of both layers and inter-laminar connections. To take into account these degradations, the composite laminate is modeled at a meso scale as a stacking of homogeneous layers connected by interfaces. Layers and interfaces may be damaged. Both onset of delamination and its propagation on a short distance are predicted. Two applications are presented, the numerical simulations are compared with experimental results: (i) delamination in the vicinity of a straight edge of a specimen under tension or compression, (ii) delamination near the hole of a perforated plate under tension.
\end{abstract}

\section{INTRODUCTION}

Delamination is a phenomenon of degradation of composite laminates that may lead structures to failure or that may reduce their stiffness and strength. This phenomenon generally occurs in edge zones of laminate structures due to possible out-of-plane stresses in these zones. The analysis of this phenomenon is commonly divided into the study of initiation of delamination and the analysis of propagation of an already delaminated area.

For the initiation prediction, computation methods of elastic free edge effects (Engrand, 1981; Dong and Goetschel, 1982; Bar-Yoseph, 1983; Dumontet, 1986) are used to evaluate the tendency of different stacking sequences to delaminate. Delamination criteria based upon elastic stresses have been proposed (Kim and Soni, 1984 and 1986; Lagace and Brewer, 1988). They consist in the comparison of the "average stress" or the "point stress" at a characteristic distance with a limit value. An important experimental procedure is necessary for the determination of the characteristic parameters. The propagation of an existing delaminated area is generally carried out by use of fracture mechanics (Wang, 1983; Jurf and Pipes, 1983; Wang, 1989).

In this paper we present the application of damage mechanics for the prediction of delamination of carbon-epoxy laminates under static loading. Degradations of layers and of connections between layers are introduced. Both onset of delamination and its propagation on a short distance are predicted.

In carbon-epoxy laminates, such as T300-914, the main types of damage that may occur are:

- progressive transverse matrix cracking,

- brittle fracture of fibers,

- debonding of fiber-matrix interface,

- debonding of adjacent layers (delamination).

The damage mechanics of composite laminates is the modeling of the degradation phenomena at a structural analysis scale. At this scale, the laminate is modeled as a stacking of homogeneous layers connected by interfaces. Relative variations of stiffness are the damage indicators. The progressive transverse matrix cracking, the brittle fracture of fibers and the debonding of the fiber-matrix interface are included at the single layer level. The damage state is assumed to be uniform in the layer thickness. The checking

\footnotetext{
* Also at Université de Marne-La-Vallée IUT.
}

${ }^{\dagger}$ Also at Université d'Evry-Val d'Essonne IUT. 
of the elastic with plasticity and damage model of the layer has been carried out (Ladevèze and Le Dantec, 1992; Allix et al., 1994) in collaboration with Aérospatiale company. The interface is a two-dimensional medium which connects two adjacent layers. Its mechanical behavior depends on the angle between the fiber directions of the adjacent layers. Its behavior is assumed to be elastic with damage.

Simulations of delamination have been compared with experimental results. These comparisons allow the identification of the interface behavior. We present two applications. The first one concerns the prediction of delamination near the straight edge of a laminate under tension or compression. Numerical simulations of onset of delamination are given and compared with experimental results from the literature.

The second application concerns the prediction of damage (delamination, transverse cracking, fiber rupture) near the hole of a perforated plate under tension, and its comparison with experimental results.

\section{LAMINATE MODELING}

At the meso scale, the laminate is modeled as a stacking of homogeneous layers connected by interfaces (Fig. 1). The interface is a zero thickness medium. Its part is limited in the edge zones where the stress state is three-dimensional. Because of their interactions (Herakovich, 1991), degradations of the layers and of the interfaces are introduced. The layer modeling has been achieved with Aérospatiale company for T300-914 and IM6-914 laminates (Ladevèze and Le Dantec, 1992; Allix et al., 1994).

\subsection{The elementary layer}

Let us note that 1,2 and 3 are, respectively, the fiber direction, the transverse direction and the normal to the laminate direction. The principal features of the modeling are:

- The layer behavior is homogeneous and orthotropic.

- In the fiber direction, the behavior is elastic and brittle under tension and it is non-linear elastic and brittle under compression.

- The only moduli modified by progressive degradations (matrix micro-cracking and fiber-matrix debonding) are the transverse and shear moduli $E_{2}$ and $G_{12}$. The other independent elastic characteristics remain constant. This is confirmed by experimental observations.

- There is no damage under transverse compression.

- To describe inelastic phenomena, a plasticity-like model with an isotropic hardening is used. There is no plasticity along the fiber direction. Plasticity and damage are coupled.

- In order to get damage modeling to agree with the classical fracture mechanics, a meso modeling is used, i.e. the damage state is assumed to be uniform in the layer thickness. In addition, delay damage modeling is introduced.

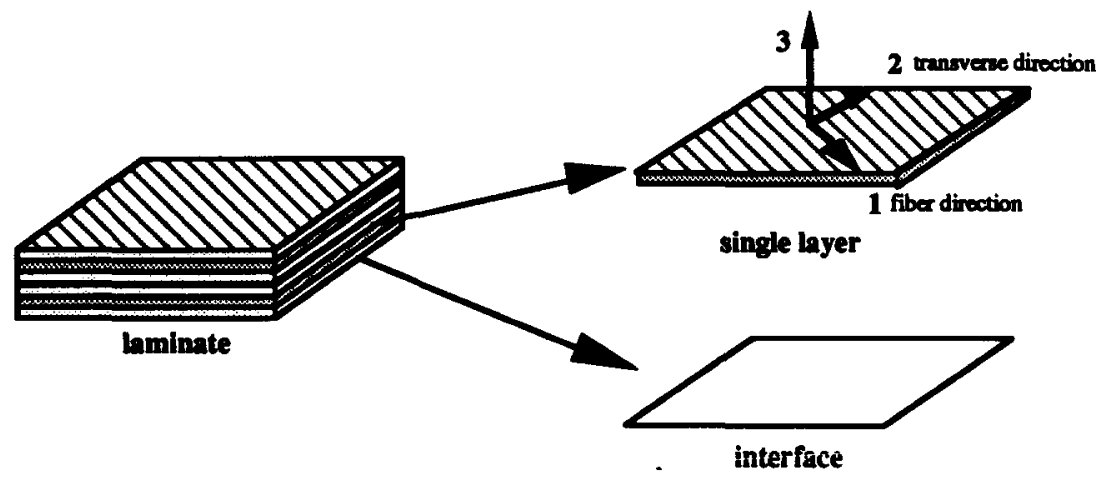

Fig. 1. Laminate modeling. 
The strain energy under plane stress assumption is:

$$
\begin{aligned}
E_{D}= & \frac{1}{2}\left[\frac{\left\langle\sigma_{11}\right\rangle_{+}^{2}}{E_{1}^{0}}+\frac{\varphi\left(\left\langle-\sigma_{11}\right\rangle_{+}\right)}{E_{1}^{0}}-\left(\frac{v_{12}^{2}}{E_{1}^{0}}+\frac{\nu_{21}^{2}}{E_{2}^{0}}\right) \sigma_{11} \sigma_{22}\right. \\
& \left.+\frac{\left\langle\sigma_{22}\right\rangle_{+}^{2}}{\left(1-d^{\prime}\right) E_{2}^{0}}+\frac{\left\langle\sigma_{22}\right\rangle_{+}^{2}}{E_{2}^{0}}+\frac{\sigma_{12}^{2}}{(1-d) G_{12}^{0}}\right] .
\end{aligned}
$$

\langle\rangle$_{+}$denotes the positive part, the superscript ${ }^{0}$ is relative to the initial moduli. $d$ and $d^{\prime}$ are two scalar damage variables. $\varphi$ is a function that takes into account the non-linear elastic behavior under compression.

2.1.1 Damage evolution. It is assumed that damage evolution of $d$ and $d^{\prime}$ is governed by the conjugate variables $Y_{d}$ and $Y_{d^{\prime}}$.

$$
Y_{d}=\left.\frac{\partial\left\langle\left\langle E_{D}\right\rangle\right\rangle}{\partial d}\right|_{\sigma}=\frac{1}{2} \frac{\left\langle\left\langle\sigma_{12}^{2}\right\rangle\right\rangle}{(1-d)^{2} G_{12}^{0}} ; \quad Y_{d^{\prime}}=\left.\frac{\partial\left\langle\left\langle E_{D}\right\rangle\right\rangle}{\partial d^{\prime}}\right|_{\sigma}=\frac{1}{2} \frac{\left\langle\left\langle\sigma_{22}^{2}\right\rangle\right\rangle}{\left(1-d^{\prime}\right)^{2} E_{2}^{0}} .
$$

《\) denotes the mean value in the ply thickness. The two damage evolutions are assumed to be coupled by: $Y=Y_{d}+b Y_{d^{\prime}}$.

The damage evolution law is:

$$
\begin{array}{ll}
\dot{d}=k\left\langle\frac{Y^{1 / 2}-Y_{0}^{1 / 2}}{Y_{c}^{1 / 2}}-d\right\rangle_{+}^{n} & \text { if } \quad d<1 ; d=1 \text { otherwise } \\
\dot{d}^{\prime}=b^{\prime} \dot{d}+k^{\prime}\left\langle\frac{Y_{d^{\prime}}^{1 / 2}-Y_{0}^{\prime 1 / 2}}{Y_{c}^{\prime 1 / 2}}-d^{\prime}\right\rangle_{+}^{n^{\prime}} & \text { if } \quad d^{\prime}<1 \text { and } Y_{d^{\prime}}<Y_{c^{\prime}} ; \quad d^{\prime}=1 \text { otherwise. }
\end{array}
$$

$Y_{0}, Y_{c}, Y_{c^{\prime}}$ and $b^{\prime}$ are characteristic parameters of the single layer. $Y_{0}, Y_{c}$ and $b^{\prime}$ are relative to a progressive damage mode while $Y_{c^{\prime}}$ is relative to the brittle rupture of the fiber-matrix interface. The influence of the $k, k^{\prime}, n$ and $n^{\prime}$ material parameters is significant in the case of large rates of damage.

\subsection{The interface}

The interface ensures displacement and traction transfer between plies. It is assumed to be elastic with damage. An interface with damage has also been used recently (Schellekens, 1991) for delamination simulation. The interface modeling we propose has been presented by Allix and Ladevèze (1992).

The principal features of the interface are listed below:

- Its behavior is assumed to be orthotropic (Fig. 2): the influence of the adjacent layers on the interface damage behavior is taken into account through the fiber direction bisectors that are supposed to be the directions of orthotropy. The constitutive law links the normal stresses to the jump of displacement between layers, its expression in elasticity is:

$$
\left(\begin{array}{c}
\sigma_{13} \\
\sigma_{23} \\
\sigma_{33}
\end{array}\right)=\left(\begin{array}{ccc}
k_{1}^{0} & 0 & 0 \\
0 & k_{2}^{0} & 0 \\
0 & 0 & k_{3}^{0}
\end{array}\right)\left(\begin{array}{l}
{\left[u_{1}\right]} \\
{\left[u_{2}\right]} \\
{\left[u_{3}\right]}
\end{array}\right)\left\{\begin{array}{l}
{[U]=U^{+}-U^{-}=\left[u_{1}\right] N_{1}+\left[u_{2}\right] N_{2}+\left[u_{3}\right] N_{3}} \\
\text { jump of displacement between the }+ \text { and - layers. }
\end{array}\right.
$$

Note the particular cases: $\begin{cases}k_{1}^{0}=k_{2}^{0}=k_{3}^{0}=0 & \text { complete debonding between layers } \\ k_{1}^{0}=k_{2}^{0}=k_{3}^{0}=\infty & \text { perfect bonding. }\end{cases}$

This kind of modeling has already been used (Léné and Léguillon, 1981) for the fiber-matrix modeling.

The interface can be considered as a rich resin zone of weak thickness $e_{I}$ compared 


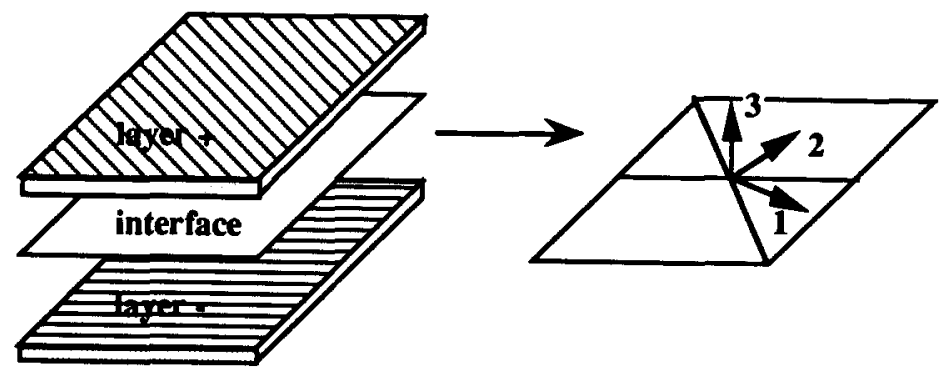

Fig. 2. Interface.

with the layer thickness $e_{C}$ (Allix and Ladevèze, 1992). By this analogy and by considering that $e_{I} / e_{C} \ll 1$, an approximation of stiffness values can be given by:

$k_{1}^{0}=\frac{2 G_{13}}{e_{I}} ; \quad k_{2}^{0}=\frac{2 G_{23}}{e_{I}} ; \quad k_{3}^{0}=\frac{E_{3}}{e_{I}} \quad G_{13}, G_{23}, E_{3}$ : elastic moduli of the rich resin zone.

They can be chosen equal to the homogenised layer moduli.

The opening mode along direction 3 corresponds to the classical fracture mechanics mode I. Displacements along directions 1 and 2 correspond to modes II and III.

- Damage is unilateral in direction 3: for the normal to the shell direction (mode I), there is no damage under compression.

- The interface behavior is assumed to be elastic with damage: that is, initially, an inelastic behavior can also be introduced (Allix and Ladevèze, 1992).

The thermodynamical potential is the strain energy (there is no contribution of the plane stresses):

$$
E_{D}=\frac{1}{2}\left[\frac{\left\langle-\sigma_{33}\right\rangle_{+}^{2}}{k_{3}^{0}}+\frac{\left\langle\sigma_{33}\right\rangle_{+}^{2}}{k_{3}^{0}\left(1-d_{3}\right)}+\frac{\sigma_{31}^{2}}{k_{1}^{0}\left(1-d_{1}\right)}+\frac{\sigma_{32}^{2}}{k_{2}^{0}\left(1-d_{2}\right)}\right] .
$$

$\langle\cdot\rangle_{+}$denotes the positive part. The variables $Y_{d i}$, which are similar to the energy release rate introduced in Fracture Mechanics, are conjugated to $d_{i}: Y_{d i}=\partial E_{D} / \partial d_{i}$

$$
Y_{d 1}=\frac{1}{2} \frac{\sigma_{31}^{2}}{k_{1}^{0}\left(1-d_{1}\right)^{2}} ; \quad Y_{d 2}=\frac{1}{2} \frac{\sigma_{32}^{2}}{k_{2}^{0}\left(1-d_{2}\right)^{2}} ; \quad Y_{d 3}=\frac{1}{2} \frac{\left\langle\sigma_{33}\right\rangle_{+}^{2}}{k_{3}^{0}\left(1-d_{3}\right)^{2}}
$$

2.2.1. A first choice for the damage evolution law. Damage evolution is assumed to be governed by:

$$
\underline{Y}=\left.\sup \right|_{\tau \leq t}\left(Y_{d 3}+\gamma_{1} Y_{d 1}+\gamma_{2} Y_{d 2}\right) \quad \gamma_{1}, \gamma_{2} \text { coupling factors }
$$

The damage evolution law is:

$$
\left\{\begin{array}{llll}
d_{3}=w(\underline{Y}) & \text { if } \quad d_{3}<1 & & d_{3}=1 \text { otherwise } \\
d_{1}=\gamma_{1} w(\underline{Y}) & \text { if } \quad d_{1}<1 \text { and } d_{3}<1 & d_{1}=1 \text { otherwise } \\
d_{2}=\gamma_{2} w(\underline{Y}) & \text { if } \quad d_{2}<1 \text { and } d_{3}<1 & d_{2}=1 \text { otherwise }
\end{array}\right.
$$

with

$$
w(\underline{Y})=\frac{\left\langle\underline{Y}-Y_{0}\right\rangle_{+}^{n}}{\left(Y_{c}-Y_{0}\right)^{n}}
$$

$\gamma_{1}, \gamma_{2}, Y_{0}$ (threshold energy), $Y_{c}$ (critical energy), $n$ : characteristic parameters of the damage evolution law of the interface are identified by simulation of delamination tests.

2.2.2. Link with fracture mechanics. Classical tests of Fracture Mechanics (Whitney, 1989) allow the three interlaminar fracture toughnesses $G_{\text {Ic }}, G_{\text {IIc }}, G_{\text {IIIc }}$, relative to the modes I, II and III to be obtained. $G_{\text {Ic }}, G_{\text {IIc }}, G_{\text {IIIc }}$ are different (Sela and Ishaï, 1989) because of the interface orthotropy. 


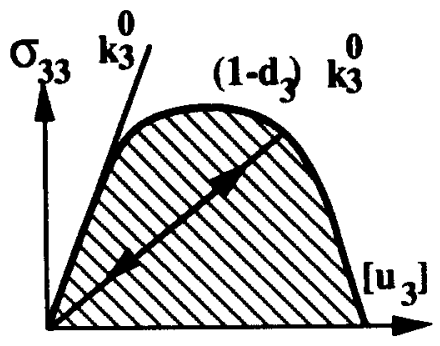

Fig. 3. Interface elastic with damage behavior (mode I traction).

$G_{c}$ can be interpreted as the necessary work per unit surface for the interface debonding. Then, it can be dependent (for instance, under pure mode $I \equiv$ mode 3 ) on the characteristic parameters of the interface modeling. $G_{I c}$ is therefore the area under the curve $\sigma_{33}-\left[u_{3}\right]$ (Fig. 3). In pure mode I one finds:

$$
G_{\mathrm{Ic}}=\int_{0}^{\left[u_{3 c}\right]} \sigma_{33} d\left[u_{3}\right] \quad \text { with } \quad\left[u_{3 c}\right]=\left.\left[u_{3}\right]\right|_{d 3=1} \Rightarrow G_{\mathrm{I} c}=Y_{0}+\frac{n}{n+1}\left(Y_{c}-Y_{0}\right)
$$

For the identification under pure modes 2 or 3 , one assumes that the 1 and 2 directions are associated with the classical modes II and III of degradation. In that case one finds:

$$
G_{\alpha c}=Y_{0}\left(\frac{2}{\gamma_{i}}-1\right)+\frac{1}{\gamma_{i}^{(1 / n+1)}}\left(1-\frac{1}{\gamma_{i}^{(n+1)}}\right)\left(Y_{c}-Y_{0}\right) \quad \text { with }\left\{\begin{array}{c}
\alpha=\text { II, III } \\
i=1,2
\end{array}\right.
$$

The above relations allow a first identification of $Y_{0}, Y_{c}, n, \gamma_{1}, \gamma_{2}$.

Note that critical energy release rates are not sufficient data because they only give information about the area under the stress-displacement curves but not on their shapes. Then, it is interesting to study the initiation of delamination, i.e. for no initial crack.

\section{SIMULATIONS OF DELAMINATION}

Because delamination phenomena are localised, the delamination analysis is carried out as a post-processor of an elastic laminate shell computation. The problem to solve is set into a zone limited to the vicinity of the structure edge.

\subsection{Near a straight edge (Daudeville and Ladevèze, 1993)}

Numerical simulations are given, in the framework of the study of delamination onset near the free edge of a specimen under tension or compression. For this approach, all the damage phenomena are now to be concentrated on the interfaces. The edge being straight, the problem to solve is a non-linear problem set into a band $B$ perpendicular to the edge. The simulations are compared with experimental results (Rodini and Eisenman, 1978; Kim and Soni, 1984) for mode I delamination (on the mid-plane interface) of a T300-5208 material under tension or compression (Fig. 4). Then, we also compared our results with experimental ones (Kim and Soni, 1986) for mixed mode of delamination of a T300-1034C under tension or compression.

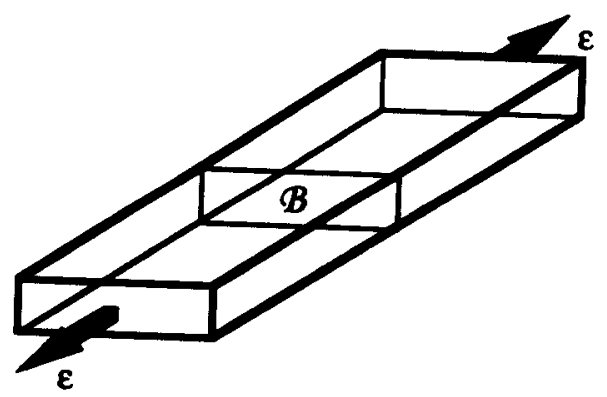

Fig. 4. Specimen under tension. 


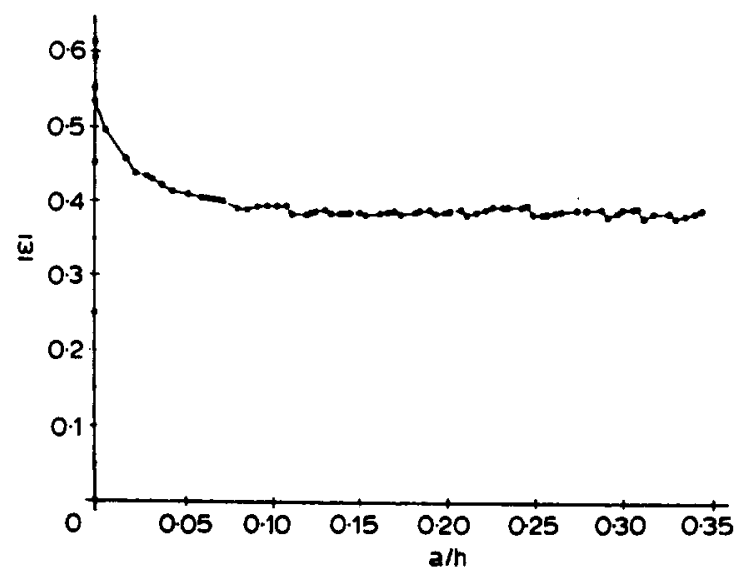

Fig. 5. Strain-crack length $(\varepsilon-a)$ curve for a $(0, \pm 45,90)$ s laminate. Delamination on the mid-plane interface.

Kim and Soni propose the same elastic moduli for both materials T300-5208 and T300-1034C.

$$
\begin{array}{ll}
E_{11}=138000 \mathrm{MPa} & G_{12}=G_{13}=G_{23}=5500 \mathrm{MPa} \\
E_{22}=E_{33}=9700 \mathrm{MPa} & v_{12}=v_{13}=0.3, v_{23}=0.6
\end{array}
$$

We have chosen $Y_{0}, n$ and the interface stiffnesses by the analogy with a film of resin:

$$
Y_{0}=0 ; n=0.5 ; e_{\mathrm{I}}=\frac{e_{\mathrm{C}}}{5}
$$

$Y_{c}$ is identified on the $( \pm 45,0,90)$ s stacking sequence, $\gamma_{1}$ and $\gamma_{2}$ are identified such that results for a mixed mode of delamination are quite good.

Figure 5 gives the strain versus the crack length for a $(0, \pm 45,90)$ s specimen under tension. Tables 1 and 2 present the comparison with other stacking sequences. The calculated strain $\varepsilon_{\text {cal }}$ in the tables corresponds to the maximum of strain-crack length curve.

Table 1. Mode I delamination

\begin{tabular}{lll}
\hline Laminate & $\varepsilon_{\text {exp }}$ & $\varepsilon_{\text {cal }}$ \\
\hline$( \pm 45,0,90) \mathrm{s}$ & 0.53 & 0.53 \\
$\left( \pm 45_{2}, 0_{2}, 90_{2}\right) \mathrm{s}$ & 0.45 & 0.38 \\
$\left( \pm 45_{3}, 0_{3}, 90_{3}\right) \mathrm{s}$ & 0.36 & 0.32 \\
$(0, \pm 45,90) \mathrm{s}$ & 0.66 & 0.61 \\
$(45,0,-45,90) \mathrm{s}$ & 0.54 & 0.57 \\
$( \pm 30,90) \mathrm{s}$ & 0.39 & 0.43 \\
$\left( \pm 30_{2}, 90_{2}\right) \mathrm{s}$ & 0.36 & 0.39 \\
\hline
\end{tabular}

Table 2. Mixed mode delamination

\begin{tabular}{llll}
\hline Laminate & Interface & $\varepsilon_{\text {exp }}$ & $\varepsilon_{\text {cal }}$ \\
\hline$( \pm 45,90,0) \mathrm{s}$ & 90,0 & +0.71 & +0.77 \\
$\left(90_{4}, \pm 30_{4}\right) \mathrm{s}$ & $30,-30$ & -0.27 & -0.28 \\
$\left(90_{4}, \pm 15_{4}\right) \mathrm{s}$ & $15,-15$ & -0.34 & -0.36 \\
$\left(0_{4}, \pm 30_{4}\right) \mathrm{s}$ & $30,-30$ & -0.35 & -0.40 \\
$\left(0_{2}, \pm 15_{2}\right) \mathrm{s}$ & $15,-15$ & -0.51 & -0.60 \\
$\left(0_{4}, \pm 15_{4}\right) \mathrm{s}$ & $15,-15$ & -0.35 & -0.45 \\
$\left(0_{4}, \pm 15_{4}\right) \mathrm{s}$ & $15,-15$ & +0.57 & +0.50 \\
\hline
\end{tabular}




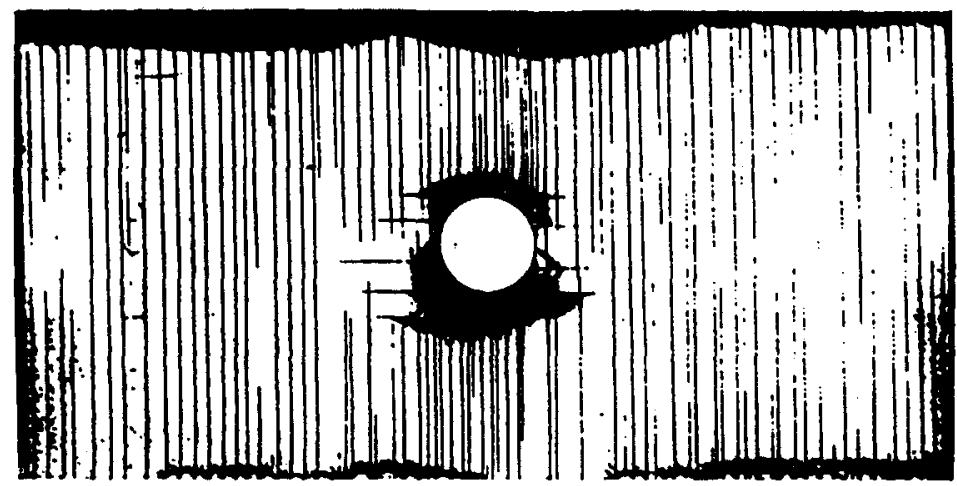

Fig. 6. $\left(0_{2}, \pm 45_{2}, 90_{2}\right) s$ plate at failure.

\subsection{Near the hole of a perforated plate (Allix and Ladevèze, 1992)}

The problem to solve is non-linear and three-dimensional. We present the analysis of delamination of a $\left(0_{2}, \pm 45_{2}, 90_{2}\right) \mathrm{s}$ T300-914 laminate under tension along the $0^{\circ}$ direction. The plate dimensions are $100 \mathrm{~mm} \times 50 \mathrm{~mm}$ and the hole diameter is $20 \mathrm{~mm}$. Both layer damage and interface damage are now taken into account. Simulation results are compared with experimental ones from Aérospatiale (Trallero, 1991). This example is very interesting because the onset of delamination does not lead to specimen failure, which allows one to follow the delamination evolution. Delamination initiation occurs between $60 \%$ and $75 \%$ of the failure load on the $0 /-45$ and $-45 / 45$ interfaces. Figure 6 presents the damaged zones just before failure (X-radiography). A diffused damage is also seen, which is interpreted through the simulation as matrix micro-cracking in the 90 , 45 and -45 plies. For the experimental level of failure, the simulation predicts the fiber breaking in the $0^{\circ}$ layer. Figures 7 and 8 show the delaminated areas that are simulated on the $0 /-45$ and $-45 / 45$ interfaces. Dark zones correspond to a total delamination $\left(d_{3}=1\right)$.

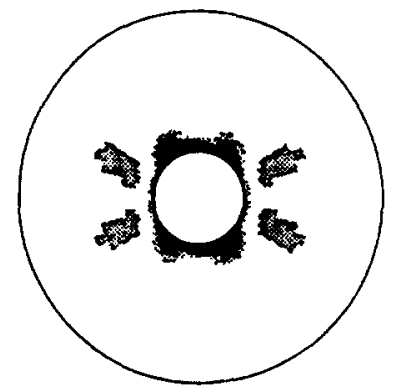

Fig. 7. Delamination on the $0 /-45$ interface.

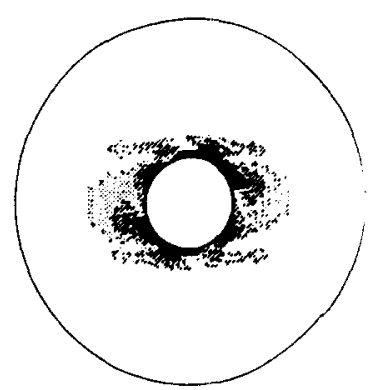

Fig. 8. Delamination on the $-45 / 45$ interface. 


\section{CONCLUSION}

In this paper, two applications of a meso-modeling of damage are presented. Damage mechanics enable the prediction of delamination initiation and propagation with a single model. That meso-modeling describes the laminate as a stacking of homogeneous layers connected by interfaces. Comparisons between numerical simulations and experimental results of delamination of a specimen under tension or compression, and a holed plate, allowed the identification of characteristic parameters of the interface. These first comparisons are encouraging on both qualitative (prediction of damaged areas) and quantitative (prediction of onset strains) points of view, in spite of a single model for every kind of interface. A possible dependence of interface characteristic parameters on the relative angle between layers must be studied.

\section{REFERENCES}

Allix, O. (1992). Damage analysis of delamination around a hole. In New Advances in Computational Structure Mechanics, (Ladevèze and Zienkiewicz), pp. 411-421. Elsevier Science Publishers, Amsterdam, Edited by Oxford.

Allix, O. and Ladevèze, P. (1992). Interlaminar interface modeling for the prediction of delamination. Compos. Struct. 22, 235-242.

Allix, O. Ladevèze, P. and Vittecoq, E. (1994). Modeling and identification of the mechanical behavior of composite laminates in compression. Comp. Sci. Tech. 51(1), 35-42.

Bar-Yoseph, P. (1983). On the accuracy of interlaminar stress calculation in laminated plates. Comp. Meth. Appl. Mech. Engng, 36, 309-329.

Daudeville, L. and Ladevèze, P. (1993). A damage mechanics tool for laminate delamination. Compos. Struct. 25, 547-555.

Dong, S. B. and Goetschel, D. B. (1982). Edge effects in laminated composites. J. Appl. Mech. 49, 129-135.

Dumontet, H. (1986). Study of a boundary layer problem in elastic composite materials. $M^{2}$ AN 20, 265-286.

Engrand, D. (1981). A boundary layer approach to the calculation of transverse stresses along the free edges of a symmetric laminated plate of arbitrary width under in plane loading. Compos. Struct. 13, 247-261.

Herakovich, C. T. (1991). Edge effects and delamination failures. J. Strain. Analysis. 26, 6260-6270.

Jurf, R. A. and Pipes, R. B. (1983). Interlaminar fracture of composite materials. J. Comp. Mater. 16, 386-394.

Kim, R. Y. and Soni, S. R. (1984). Experimental and analytical studies on the onset of delamination in laminated composites. J. Comp. Mater. 18, 70-76.

Kim, R. Y. and Soni, S. R. (1986). Delamination of composite laminates stimulated by interlaminar shear. ASTM-STP 893, 286-307.

Ladevèze, P. and Le Dantec, E. (1992). Damage modeling of the elementary ply for laminated composites. Comp. Sci. Technol. 43(3), 257-267.

Lagace, P. A. and Brewer, J. C. (1988). Quadratic stress criterion for initiation of delamination. J. Comp. Mater. 22, 1141-1155.

Léné, F. and Léguillon, D. (1981). Etude de l'influence d'un glissement entre les constituants d'un matériau composite sur ses coefficients de comportement effectifs. Journal de Mécanique 20, 509-536.

Rodini, B. T. and Eisenman, J. R. (1978). An analytical and experimental investigation of edge delamination in composite laminates. Proc. 4th Conf. Fibrous Comp., San Diego, edited by Lenoe, Oplinger and Burke, pp. 441-457.

Schellekens, J. C. J. and De Borst, R. (1991). Numerical simulation of free edge delamination in graphite epoxy specimen under uniaxial extension. Sixth Int.Conf. Compos. Struct., Paisley, edited by I. H. Marshall, pp. 647-657. Elsevier Science Publishers, Amsterdam, London.

Sela, N. and Ishai, O. (1989). Interlaminar fracture toughness and toughening of laminated composite materials: a review. Composites 20, 423-435.

Trallero, D. (1991). Etude expérimentale et numérique de l'endommagement de structures percées en matériaux stratifiés, Mémoire d'Ingénieur CNAM.

Wang, A. S. D. (1989). Fracture analysis of interlaminar cracking. Interlaminar Response of Composite Materials, Composite Material Series (Edited by N. J. Pagano), Vol. 5, pp. 69-109. Elsevier Science Publishers. Amsterdam, Oxford.

Wang, S. S. (1983). Fracture mechanics for delamination problems in composite laminates. J. Comp. Mater. 16, 386-394.

Whitney, J. M. (1989), Experimental characterization of delamination fracture. In Interlaminar Response of Composite Materials, Composite Material Series (Edited by N. J. Pagano), Vol. 5, pp. 161-250. Elsevier Science Publishers, Amsterdam, Oxford. 